\title{
Development of cutaneous microflora in premature neonates
}

\author{
N Keyworth, M R Millar, K T Holland
}

\begin{abstract}
Coagulase-negative staphylococci (C-NS) are a frequent cause of bacteraemia in premature neonates. It is likely that the strains of C-NS causing bacterial sepsis in premature neonates have their origin on the patient's skin surface. We have studied the quantitative development of the skin microflora at eight sites on premature neonates.
\end{abstract}

A swab wash method was used to sample and enumerate the cutaneous microflora of premature neonates admitted to an intensive care unit with respiratory distress syndrome. The numbers of bacteria present on the skin increased rapidly by 100-fold in the first week of life. The species of C-NS found on neonatal skin were similar to those found on adult skin. However, the bacterial population was $10^{3}$ lower by comparison. There was considerable variation in numbers of bacteria and in the proportion resistant to antibiotics from day to day. There appeared to be no association between antibiotic usage and the proportion of isolates resistant to antibiotics, although the resident bacteria were in many cases resistant to a variety of antibiotics. C-NS were isolated from $92 \%$ of samples from which bacteria were isolated. Staphylococcus epidermidis was found at all sites and accounted for $82 \%$ of each colonial type of staphylococcus isolated. Other organisms isolated included Propionibacterium sp, $\alpha$-haemolytic streptococci, aerobic spore-bearing bacilli, aerobic coryneforms, Candida albicans, Klebsiella oxytoca, Pityrosporum sp, Klebsiella pneumoniae, and Escherichia coli.

The results of this study suggest that the skin of premature neonates is colonised with antibiotic resistant C-NS during the first week of life and that the chance of contamination of an intravascular catheter at insertion increases during this period.

Coagulase-negative staphylococci (C-NS) are frequent isolates from blood cultures of premature neonates. ${ }^{12}$ Episodes of bacteraemia with C-NS are usually associated with intravascular catheters ${ }^{3}$ and diagnosed after the first week of postnatal life. ${ }^{2}$ Intravascular catheters predispose to bacteraemia with C-NS both by providing a break in the cutaneous barrier and by acting as a foreign body, reducing local resistance to infection. Quantitative blood culture techniques have shown that numbers of C-NS present in blood of premature neonates with bacteraemia may exceed 1000 colony forming units (cfu)/ml. ${ }^{45}$ Defective opsonisation and phagocytosis may allow these large numbers of C-NS to circulate in the blood. ${ }^{6}$

The strains of C-NS most frequently associated with neonatal infection are often resistant to a wide range of antimicrobials. ${ }^{7}$ Antibiotic resistant C-NS spread from patient to patient via the hands of nursing and medical staff, leading to colonisation of the skin of premature neonates in intensive care units within the first week of life. The numbers of bacteria on the skin surface will determine the probability and degree of contamination of the catheter at the time of insertion. Local immune defences are more likely to be overcome when large numbers of bacteria are present on the skin at the catheter insertion site.

Qualitative studies suggest that the skin of premature neonates becomes colonised with C-NS within the first few days of life. ${ }^{8}$ However, the quantitative development of the cutaneous microflora has not been studied. This is due in part to unsuitability of methods of skin sampling which have been developed primarily for use on adults and may damage the skin of neonates. In this study we have used a non-traumatic swab wash method $^{9}$ to study the quantitative and qualitative changes in the bacterial flora and in the level and pattern of antibiotic resistance of these bacteria. This method of sampling gives comparable results in full term neonates and adults to those obtained with the scrub wash method of Williamson and Kligman. ${ }^{10}$

\section{Patients and methods}

\section{SAMPLING OF CUTANEOUS MICROFLORA}

Nine premature neonates admitted to the Peter Congdon Regional Neonatal Unit, The General Infirmary, Leeds, between 25 January and 21 April 1990 with respiratory distress syndrome were sampled. The gestational age range was 25-32 weeks with birth weight ranging from 680 to $1920 \mathrm{~g}$. Neonates were selected on the basis of prematurity only $(<33$ weeks' gestation). The following sites were sampled using a swab wash method': forehead, chest, periumbilical region, back, upper and lower arm, and upper and lower leg. Neonates were sampled within six hours of birth, on days $3,5,7$, and weekly thereafter. Local ethical committee approval was 
obtained for this study. Informed consent was obtained from the parents of each infant sampled.

\section{QUANTITATION OF MICRO-ORGANISMS IN WASH} FLUIDS

Samples were serially diluted 10 -fold in half strength wash fluid. Each dilution was vigorously mixed, and $100 \mu \mathrm{l}$ volumes of diluted and undiluted wash fluid were spread over the surface of the following selective and nonselective media: heated $5 \%(\mathrm{v} / \mathrm{v})$ horse blood agar (CM 55: Oxoid) incubated at $37^{\circ} \mathrm{C}$ in air for four days; Sabouraud dextrose agar (Oxoid CM 41) with chloramphenicol $(50 \mathrm{mg}$ 1) incubated at $37^{\circ} \mathrm{C}$ in air for seven days; $5 \%$ (v/v) horse blood agar with neomycin (final concentration $28 \mathrm{mg} / \mathrm{l}$ ) incubated at $27^{\circ} \mathrm{C}$ anaerobically for seven days; aerobic coryneform agar with and without furazolidone (6 $\mathrm{mg} / \mathrm{l}$ ) incubated at $37^{\circ} \mathrm{C}$ aerobically for two days ${ }^{11}$; reinforced clostridial agar with and without furazolidone incubated at $37^{\circ} \mathrm{C}$ for seven days anaerobically ${ }^{11}$; and pityrosporum agar ${ }^{12}$ incubated at $34^{\circ} \mathrm{C}$ aerobically in a moist chamber for 14 days. After incubation, colonies were enumerated with a colony counter (Gallenkamp CNW325010X) to give a total viable count expressed as $\mathrm{cfu} / \mathrm{cm}^{2}$ and then logarithmically transformed.

\section{IDENTIFICATION OF ORGANISMS}

Preliminary identification of staphylococcal species was based on colonial morphology, positive catalase test, slide coagulase test, and plate DNAse test. ${ }^{13}$ One colony of each colonial type was identified as to species by the scheme of Kloos and Schleifer. ${ }^{14}$ Catalase negative Gram positive cocci were identified on the basis of haemolysis and growth at $45^{\circ} \mathrm{C}$. Aerobic coryneform bacteria were infrequently isolated and were not differentiated further. Gram negative rods were identified using the API 20E enterobacteriaceae biochemical typing system. Yeasts growing on pityrosporum agar were confirmed as Malassezia furfur species by colonial appearance and microscopic morphology. Yeasts isolated on Sabouraud dextrose agar were tested for the ability to form germ tubes (isolates were examined microscopically for germ tube formation after incubation for two hours in horse serum). Germ tube positive isolates were identified as Candida albicans, whereas germ tube negative isolates were grouped as Candida sp. Propionibacteria were identified on the basis of colony and Gram stain morphology and classified as to species as described by Holland. ${ }^{15}$

PROPORTION OF CUTANEOUS FLORA RESISTANT TO ANTIBIOTICS

In order to determine directly the sensitivity of bacterial skin isolates to six antimicrobial agents, antibiotics were incorporated at the following concentrations into Isosensitest (Oxoid) agar plates:vancomycin $4.0 \mathrm{mg} / \mathrm{l}$, gentamicin $0.5 \mathrm{mg} / \mathrm{l}$, erythromycin $2.0 \mathrm{mg} / \mathrm{l}$, methicillin $2.0 \mathrm{mg} / \mathrm{l}$, penicillin $1.0 \mathrm{mg} / \mathrm{l}$, and chloramphenicol $32 \cdot 0 \mathrm{mg} /$.
Then $100 \mu$ of swab wash fluid was plated onto each media. The numbers of colonies growing on each of the antibiotic-containing plates, with reference to colony counts on media containing no antibiotics, was used to calculate the percentage of the bacterial population that was sensitive to each antibiotic.

DETECTION OF ANTIMICROBIAL SUBSTANCES IN SAMPLE FLUIDS

The presence of antimicrobial substances in skin wash fluid samples was detected by pipetting $100 \mu \mathrm{l}$ of the sample into wells previously cut in Isosensitest agar plates that had been surface seeded with Oxford staphylococcus (NCTC 6571). Detection of gentamicin in wash fluid samples was by colorimetric assay using a TDX antimicrobial assay machine (Abbott).

HAND IMPRESSION PLATES

At each sampling a horse blood agar (Oxoid) hand impression plate was taken of the attendant nurses' finger pads and nail margins.

\section{Results}

The swab wash method allowed repeated sampling without trauma to fragile neonatal skin. However, daily sampling was not performed in case this disrupted normal skin flora development. No adverse effects on neonatal skin were noticed during the study period.

Acquisition of skin flora followed the same pattern in the four neonates born vaginally after normal labour and in the five neonates born by caesarean section. A surprising observation was the lack of vaginal flora and enterobacteriaceae in early swab wash samples from infants born vaginally.

Bacteria were detectable on the skin surface within six hours of birth. The most common bacterial isolates during the study were C-NS. C-NS were isolated from $92 \%$ of samples containing bacteria. Organisms other than CNS were infrequently isolated from single sites. These organisms were probably not part of the developing microflora and at no time did they replace staphylococci as predominant flora. Other organisms isolated from swab wash samples were: Propionibacterium sp, $\alpha$ haemolytic streptococci, aerobic spore bearing bacilli, aerobic coryneforms, $C$ albicans, Klebsiella oxytoca, Pityrosporum sp, Klebsiella pneumoniae, and Escherichia coli.

Numbers of staphylococci present at the skin sites sampled rose rapidly during the first 7 days of postnatal life (fig 1). Acquisition of cutaneous C-NS in neonates of $\leq 28$ weeks' and $>28$ weeks' gestational age followed a similar pattern.

During the study 2548 staphylococcal isolates were selected by colonial morphology for species identification. Staphylococcus epidermidis was found at all sites and accounted for $82 \%$ of the total colonial types of staphylococci. Other species of staphylococcus isolated were: $S$ warnerii $4 \cdot 2 \%, S$ haemolyticus $14 \cdot 1 \%, S$ aureus $2 \cdot 4 \%, S$ saprophyticus $1.6 \%$, $S$ hominis 1 


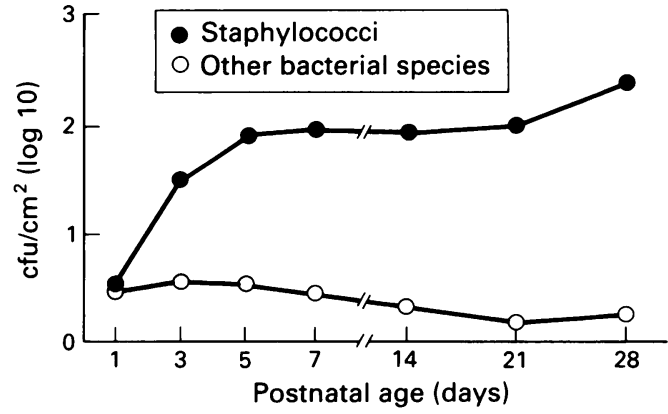

Figure 1 Quantitative changes in cutaneous microflora with postnatal age. Measurements are logarithmically transformed and taken at eight sites in nine neonates.

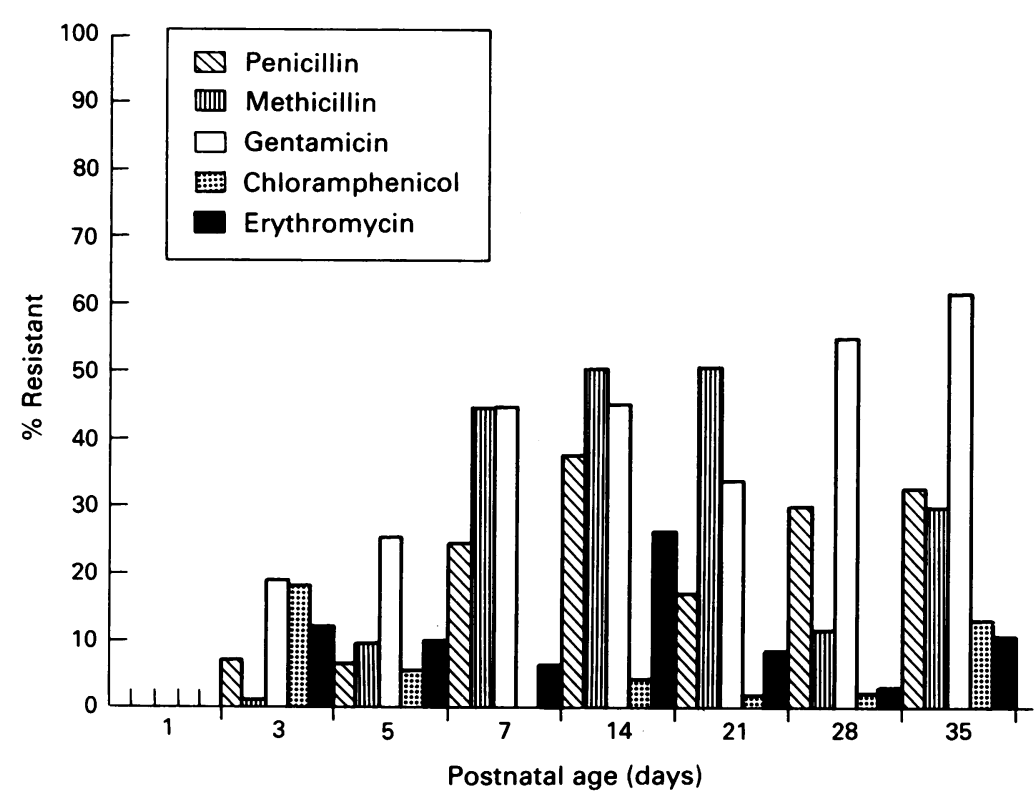

Figure 2 Changes in percentage of cutaneous staphylococci resistant to antibiotics with postnatal age. Measurements taken in all neonates at all sites.

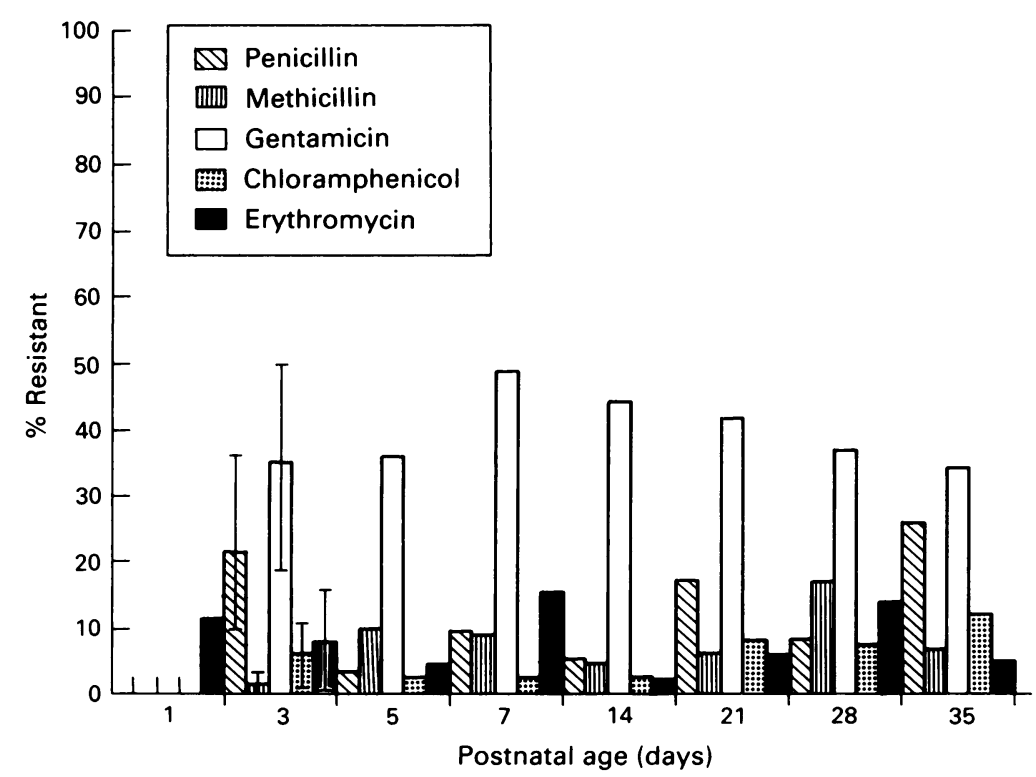

Figure 3 Changes in percentage of cutaneous staphylococci resistant to antibiotics with postnatal age. Measurements taken on the forearm in all neonates showing SD on day 3.
$0 \cdot 8 \%, S$ hominis $20 \cdot 7 \%$, $S$ haemolyticus 2 $0.6 \%, S$ xylosis $10.5 \%, S$ capitis $0.4 \%, S$ cohnii $0.3 \%, S$ xylosis $20 \cdot 2 \%$, and $S$ simulans $0.03 \%$ and micrococcus $2.0 \%$. This rapid development of cutaneous microflora was unstable, with considerable day to day variation in numbers $\left(0-1 \times 10^{3} \mathrm{cfu} / \mathrm{cm}^{2}\right)$ and in species present. A similar pattern of variation was seen in all neonates at all sites.

The proportion of C-NS resistant to antibiotics, determined by comparison of bacterial counts of swab wash samples on media with and without added antibiotics, revealed similar day to day variation (fig 2). Antibiotic resistant C-NS were present in all neonates by the end of the first week of life. Resistance to antibiotics used on the unit was an expected finding. However, resistance to antibiotics not commonly used, such as chloramphenicol, was also observed. The development with time of the percentage of cutaneous staphylococci from the forearm that were resistant to antibiotics is shown in fig 3. The forearm was chosen as an example as it is here that peripheral intravenous catheters are often sited.

The high proportion of isolates resistant to gentamicin was a common finding in all neonates at all sites. Quantitative changes in cutaneous staphylococci and percentage resistance to antibiotics with postnatal age is shown in one premature neonate in fig 4. Antibiotic resistance of the skin microflora did not appear to be temporally related to the use of antibiotics. Generally there was a wide variation in numbers and types of organisms with no correlation of species with site.

Antimicrobial activity on neonatal skin was demonstrated in $14 \cdot 1 \%$ of 320 unselected swab wash samples. The finding of gentamicin in one swab wash fluid was probably due to gentamicin excreted in urine which contaminated the skin. A swab wash fluid exhibiting antimicrobial activity which was neutralised with the addition of $\beta$-lactamase solution was also noted. The finding of antimicrobial activity was not related to site of sampling.

The hand impression plates obtained from attending staff usually yielded C-NS, which were identified by the methods mentioned earlier. On one occasion $S$ xylosis 1 , an uncommon isolate, was detected on both neonatal skin and attendant's hand plate, suggesting transfer of organisms between the two.

\section{Discussion}

C-NS are the most frequent blood culture isolates from premature neonates in many intensive care units. Episodes of bacteraemia with C-NS are usually associated with intravascular catheters and it is likely that these devices provide the focus of infection from which bacteraemia develops. ${ }^{3}$ An association between microbial numbers on the skin and the development of cannula related infection has been postulated. ${ }^{16} S$ epidermidis is the species most often associated with neonatal infections and in this study accounted for $82 \%$ of the total colonial types isolated. Blood culture isolates 


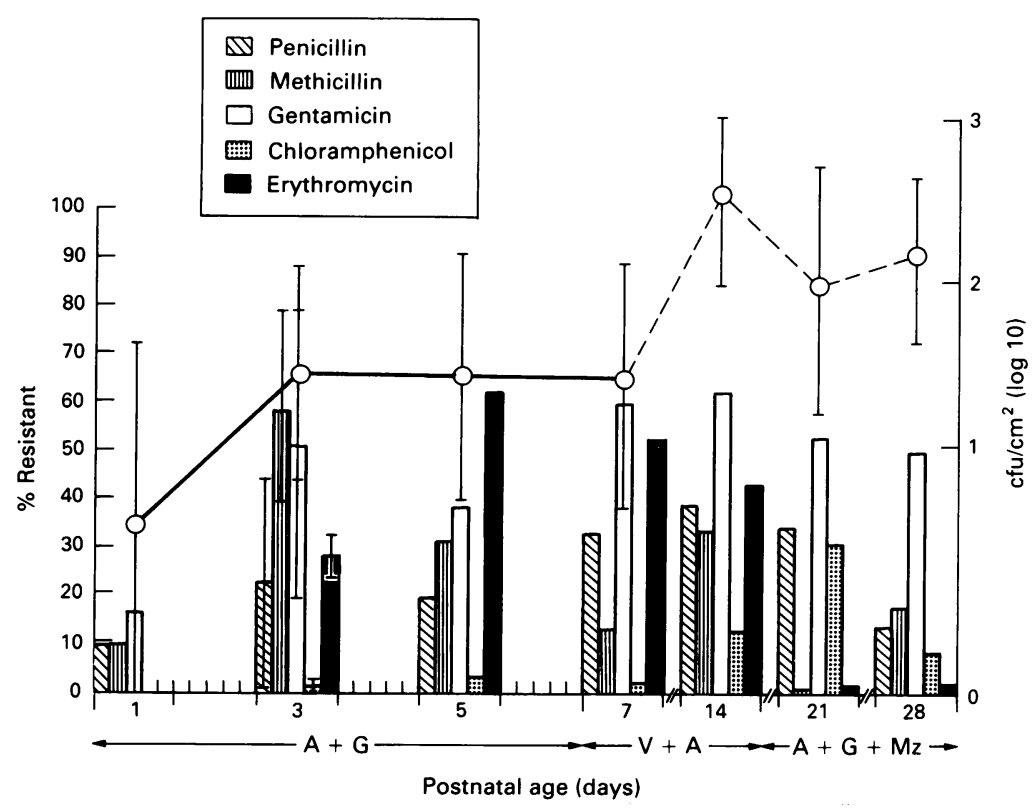

Figure 4 Quantitative changes in cutaneous staphylococci and percentage resistant to antibiotics with postnatal age. Resistance is shown in relation to treatment: ampicillin $(A)$ and gentamicin $(G)$ given to day 6 , the vancomycin $(V)$ and ampicillin, followed by ampicillin, gentamicin, and metronidazole (Mz).

of C-NS are usually resistant to a wide range of antibiotics. ${ }^{7}$ In this study antibiotic resistant C-NS were isolated from the skin of premature neonates within days of admission to the neonatal intensive care unit. ${ }^{8}$ This finding is possibly due to handling. C-NS were the most common isolates from neonatal skin in this study and $S$ epidermidis the most frequent species.

Previous studies of bacterial colonisation of neonates have concentrated on sampling the anterior nares, axilla, and umbilicus of mature neonates and have not quantified bacterial numbers. ${ }^{8}$ In this study the cutaneous microflora at eight sites were quantified, including sites used for intravascular catheter insertion. A number of factors might be expected to influence the numbers of C-NS on the skin of neonates, including nutrient availability, prolonged occlusion, environmental humidity and temperature, type of incubator, exposure to ultraviolet light (phototherapy) and antibiotics, topical disinfectants, $\mathrm{pH}$ and redox potential at colonised sites, and nursing practices. ${ }^{17}$

The flora of normal human skin in both children and adults does not vary widely from day to day and, with the exception of changes occurring at puberty, is extremely stable. This would indicate that the skin environment is also stable. In contrast, the variation and the relatively low numbers of skin microflora in this study (counts on adult skin may be $10^{4}-10^{5}$ higher $^{18}$ ) indicate that the skin of premature neonates is not supportive of a stable microbial ecosystem.

The skin environment of premature neonates appears only able to sustain low numbers of staphylococci and does not have the morphological or nutritional value to support other micro-organisms. Also, by contrast with findings in adults, there was no consistent pattern of site to site variation in bacterial numbers and there was no association of species of C-NS with specific sites. ${ }^{19}$

There was considerable variation in the antibiotic sensitivity pattern and biotype of isolates of C-NS obtained from the same neonate from one sampling to another, suggesting that the cutaneous microflora of premature neonates in the first month of life changes rapidly. It is likely that the microflora of premature neonates' skin is maintained in an erratic fashion by repeated inoculation due to the continual handling by attendant staff, and consists of a multiple transient flora which is reinoculated each time the neonate is handled. This hypothesis is supported by the findings of relatively few organisms, occasions when no organisms were detected at a site where organisms were previously isolated, and fluctuation in antibiotic resistance patterns not related to antibiotic use.

Possible factors preventing the stability of skin micro-organisms include the structure of the skin in the first weeks of life, ${ }^{20}$ the presence of soluble factors on the skin inhibiting the growth of micro-organisms, or desiccation. Further work may help to determine when a stable microflora is established.

Antibiotic resistant strains of C-NS are more likely to be isolated from the skin of hospital patients than from people in the community. ${ }^{21}$ Antibiotic resistant staphylococci isolated in this study may have been derived from (a) the hands of hospital staff, (b) from the bowel flora and inoculated onto the skin from faecal contamination, or (c) a combination of both.

Handling by medical and nursing staff will spread bacteria from site to site. It was not possible to correlate organisms found on the neonates' skin and hand carriage by attendant staff. Skin maturity did not appear to be a factor in acquisition of skin microflora, as development of the cutaneous microflora was similar in neonates of less than 29 weeks' gestational age to those of 29 or more weeks' gestational age. Enterobacteriaceae and yeasts were isolated infrequently, not related to any clinical signs and probably reflected intermittent hand carriage by attendant staff.

A high proportion of isolates of C-NS in this study were resistant to antibiotics including antibiotics not commonly used (such as chloramphenicol). This may be due to linkage of genes coding for resistance to antimicrobials on the same plasmid. In this study there seemed to be no association between the resistance patterns seen and the degree of resistance in the population to the antibiotics in use. Gentamicin was detected in some of the swab wash samplings, perhaps as a result of contamination of the skin with urine containing high concentrations of excreted gentamicin.

Bacteraemia with C-NS is uncommon in the first week of life. ${ }^{2}$ We have found that the numbers of C-NS present on the surface of the skin increase during the first week of life. High concentrations of C-NS present on the skin surface at the time of catheter insertion will increase the chance of catheter tip contamination and the subsequent probability 
of bacterial invasion between the catheter and the thin skin of premature neonates.

These findings highlight the need for good skin preparation with an efficient skin disinfectant before inserting intravenous catheters and, as repopulation of the skin at the entry site occurs within 18 hours, ${ }^{22}$ for site care after insertion. Good handwashing technique and minimal handling will help reduce the hand carriage and transfer of microorganisms.

1 Battisti O, Mitchison R, Davis PA. Changing blood culture isolates in a referral neonatal intensive care unit. Arch Dis Child 1981;56:775-8.

2 Freedman RM, Ingam DL, Goss I, Ehrenkranz A Warshaw JB, Baltimore RS. A half century of neonatal sepsis at York 1928-1978. Am $\mathcal{f}$ Dis Child 1981;135:140-4.

3 Noel GJ, Edelson PJ. Staphylococcus epidermidis bacteraemia in neonates: further observations and occurrence .

Kite P, Langdale V, Todd N, Millar MR, Mackay P. Direct isolation of CNS from neonatal blood samples. $\mathcal{f}$ Hosp Infect 1989;14:135-40

5 Philips SE, Bradley JS. Bacteraemia detected by lysis direct plating in a neonatal ICU. $f$ Clin Microbiol 1990;28:1-4.

6 Fleer A, Gerards LJ, Aerts P, et al. Opsonic defence to Staphylococcus epidermidis in the premature neonate. f Infect Dis 1985;152:930-7.

7 Hall RT, Hall SL, Barnes WG, et al. Characteristics of CNS from infants with bacteraemia. Pediatr Infect Dis 1987;6:388-83.

8 D'Angio CT, McGowan KL, Baumgart S, Gem JSt, Harris MC. Surface colonization with CNS in premature neonates. F Pediatr 1989;114:1029-34.
9 Keyworth N, Millar MR, Holland KT. Swab wash method for quantitation of cutaneous microflora. $\mathcal{F}$ Clin Microbiol 1990;28:941-3.

10 Williamson P, Kligman AM. A new method for the qualitative investigation of cutaneous bacteria. $\mathcal{F}$ Invest Dermatol 1965;45:498-503.

11 Cove JH, Eady EA. A note on a selective medium for the isolation of cutaneous propionibacteri. $\mathcal{f}$ Appl Bacteriol 1982;53:289-92.

12 Leeming JP, Notman FH. Improved method for isolation and enumeration of Malassizia furfur from human skin. $\Im$ Clin Microbiol 1987;25:2017-9.

13 Disalvo JW. Deoxyribonucleic acid coagulase acting micrococci. US Armed Forces M F Tech Bull 1958;9:191-6.

14 Kloos WE, Schleifer KH. Simplified scheme for routine identification of human Staphylococcus species. $f$ Clin Microbiol 1975;1:82-8.

15 Holland KT. Microbiology of acne. In: Cunliffe WJ, ed. Acne. London: Martin Dunitz, 1989:178-218

16 Snydman DR, Pober BR, Murray SA, Gorbea HF, Majka JA, Perry LK. Predictive value of surveillance skin cultures in total-parenteral-nutrition-related infection. Lancet 1982;ii: 1385-8.

17 Hartmann AA. The influence of various factors on the human resident skin flora. Semin Dermatol 1990;9: 305-8.

18 Noble WC. Microbiology of human skin. 2nd Ed. London: Lloyd Luke, 1981

19 Kloos WE, Musselwhite MS. Distribution and persistence of Staphylococcus and Micrococcus species and other aerobic bacteria on human skin. Microbiology 1975;30. $381-95$

20 Evans NJ, Rutter N. Development of the epidermidis in the newborn. Biol Neonate 1986;49:74-80.

21 Keyworth $\mathrm{N}$. The characterisation of phenotypic beta lactam resistance in coagulase negative staphylococci. Leeds: University of Leeds, 1988. (M Phil thesis.)

22 Hendley JO, Asme KM. Effect of topical antimicrobial treatment on aerobic bacteria in the stratum corneum of human skin. Antimicrob Agents Chemother 1991;35: 627-31. 